\title{
Dietary antioxidant capacity and risk of type 2 diabetes in the large prospective E3N-EPIC cohort
}

\author{
Francesca Romana Mancini ${ }^{1}$ - Aurélie Affret ${ }^{1}$ - Courtney Dow ${ }^{1} \cdot$ Beverley Balkau $^{2}$. \\ Fabrice Bonnet ${ }^{1,3} \cdot$ Marie-Christine Boutron-Ruault ${ }^{1}$. Guy Fagherazzi ${ }^{1}$
}

Received: 21 March 2017 / Accepted: 28 September 2017 / Published online: 9 November 2017

(C) Springer-Verlag GmbH Germany 2017

\begin{abstract}
Aims/hypothesis Recent evidence suggests that oxidative stress may contribute to the pathogenesis of type 2 diabetes. The diet, and especially fruit and vegetables, contains a variety of compounds with antioxidant activity, which may have cumulative/synergistic antioxidant effects. The total antioxidant capacity, an index derived from dietary intake, is a single estimate of antioxidant capacity from all dietary antioxidants. The main aim of this study was to investigate the relationship between total antioxidant capacity and risk of type 2 diabetes. Methods Among 64,223 women (mean age $52 \pm 7$ years) from the French E3N-European Prospective Investigation into Cancer and Nutrition (EPIC) cohort, 1751 women had validated type 2 diabetes during 15 years of follow-up. The total antioxidant capacity was estimated with the ferric ionreducing antioxidant power (FRAP) method. Adjusted Cox proportional hazards regression models were used to calculate HRs and 95\% CIs for the associations between total
\end{abstract}

Electronic supplementary material The online version of this article (https://doi.org/10.1007/s00125-017-4489-7) contains peer-reviewed but unedited supplementary material, which is available to authorised users.

Marie-Christine Boutron-Ruault

marie-christine.boutron@gustaveroussy.fr

1 Inserm U1018, Centre for Research in Epidemiology and Population Health (CESP) 'Health across Generations' Team, University Paris-Saclay, University Paris-Sud, Gustave Roussy, Espace Maurice Tubiana, 114 rue Edouard Vaillant, 94805 Villejuif Cedex, France

2 Inserm U1018, Centre for Research in Epidemiology and Population Health (CESP) 'Renal and cardiovascular Epidemiology' Team, University Versailles, Saint Quentin, University Paris-Sud, Villejuif, France

3 CHU Rennes, Université de Rennes 1, Department of Endocrinology, Diabetology and Nutrition, Rennes, France antioxidant capacity and type 2 diabetes risk, adjusted for potential confounders.

Results In multivariable models, higher levels of total antioxidant capacity were associated with a lower risk of type 2 diabetes. Compared with women in the lowest quintile, women in the third, fourth and fifth quintiles for total antioxidant capacity had HRs of 0.74 (95\% CI 0.63, 0.86), 0.70 (95\% CI $0.59,0.83)$ and $0.73(95 \%$ CI $0.60,0.89)$, respectively. The inverse association between total antioxidant capacity and risk of type 2 diabetes was linear up to values of $15 \mathrm{mmol} / \mathrm{day}$, after which the effect reached a plateau.

Conclusions/interpretation Our findings suggest that the total antioxidant capacity may play an important role in reducing the risk of type 2 diabetes in middle-aged women. More studies are warranted to better understand the biological mechanisms underlying this inverse association.

Keywords Diet · E3N cohort · FRAP (ferric ion-reducing antioxidant potential) $\cdot$ Risk $\cdot$ Total antioxidant capacity $\cdot$ Type 2 diabetes
Abbreviations
EPIC European Prospective Investigation into Cancer and Nutrition
FRAP Ferric ion-reducing antioxidant power
MET Metabolic equivalents
TRAP Total radical-trapping antioxidant parameter

\section{Introduction}

Type 2 diabetes is a metabolic disorder characterised by hyperglycaemia and insulin resistance [1]. The main type 2 diabetes risk factors are lifestyle related and include high BMI, 
physical inactivity and an energy-dense diet [2]. Increasing evidence from both experimental and clinical studies suggests that oxidative stress plays a major role in the pathogenesis of type 2 diabetes: abnormally high levels of free radicals and the simultaneous decline of antioxidant defence mechanisms can lead to damage of cellular organelles and enzymes, increased lipid peroxidation and development of insulin resistance [3-5]. Moreover, previous studies have highlighted how increased intake of common dietary antioxidants such as vitamin $\mathrm{E}$ and vitamin $\mathrm{C}$ increase insulin sensitivity, reduce fasting and post-meal blood glucose and improve $\mathrm{HbA}_{1 \mathrm{c}}$ levels $[6,7]$. Other authors have reported a statistically significant decrease in total antioxidant status and increase in levels of oxidative stress markers in individuals with type 2 diabetes compared with healthy individuals, and have suggested that these levels could be considered markers of early disease and predictors of type 2 diabetes complications [8].

Dietary compounds with antioxidant activity may have cumulative/synergistic antioxidant effects. The main known contributors to the overall antioxidant capacity of the diet are fruit and vegetables, wine, coffee and tea [9]. Antioxidants from the diet act in two main ways: first, preventing the generation of excess free radicals, and thus avoiding oxidative damage to the cell; and second, after damage has occurred, preventing further cell degeneration, thus alleviating the progression of conditions caused by oxidative stress [10]. Recently, an index of the total antioxidant capacity of foods, which describes the ability of food antioxidants to scavenge preformed free radicals, has been suggested as a tool for investigating the health effects of antioxidants present in mixed diets. The total antioxidant capacity concept aims to group the capacities from all dietary antioxidants into a single estimate [11]. A high total antioxidant capacity has been related to several health benefits in cohort, cross-sectional and interventional studies [12-17]. These findings support total antioxidant capacity as a promising functional descriptor of the protective effects of dietary antioxidants in different populations. To date, no study has investigated the association between total antioxidant capacity and risk of type 2 diabetes.

The purpose of this study was to investigate the relationship between total antioxidant capacity and risk of type 2 diabetes in the E3N-European Prospective Investigation into Cancer and Nutrition (EPIC) cohort, a large cohort of French women.

\section{Methods}

The E3N-EPIC study The E3N-EPIC prospective cohort was initiated in France in 1990 to study the main risk factors for cancer and severe chronic conditions in women $[18,19]$. It is the French constituent of the large EPIC study, and the EPIC sub-study devoted to diabetes: Interaction of Genetic and
Lifestyle Factors on the Incidence of Type 2 Diabetes (InterAct) [20]. The E3N-EPIC cohort includes 98,995 French women born between 1925 and 1950, insured by the Mutuelle Générale de l'Education Nationale (MGEN), a national teachers' health insurance plan. All women gave written informed consent and approval was obtained from the Commission National Informatique et Libertés (French National Commission for Data Protection and Individual Freedom).

Population for analysis Among the 74,522 women with dietary information available, women were excluded if they did not complete any questionnaire after the dietary questionnaire $(n=936)$. Women were also excluded if they had extreme values for the ratio of energy intake to required energy (i.e. they were in the first and 99th percentiles of the distribution in the population) $(n=1466)$. Basal metabolic rate (BMR), based on age, sex and weight (self-reported in $\mathrm{kg}$ ), multiplied by 1.55 was used to estimate a woman's energy intake. Finally, women with pre-existing cardiovascular disease ( $n=476)$, pre-existing diabetes $(n=1150)$ or pre-existing cancer $(n=4129)$ were excluded from the present study. Women with potential pre-existing, but not validated, diabetes (2142) were also excluded. Thus, this analysis included 64,223 women, of whom 1751 had validated type 2 diabetes diagnosed during follow-up (June 1993-December 2008).

Ascertainment of type 2 diabetes Women who potentially had diabetes were identified from two sources. First, selfreporting of diabetes, diabetes diet, diabetes drugs or hospitalisation for diabetes in follow-up questionnaires. Second, through the insurance drug reimbursement database: all women reimbursed for glucose-lowering medications at least once after January 2004 were considered as potentially having diabetes.

All women who potentially had diabetes were contacted and asked to answer a diabetes-specific questionnaire that included questions on the circumstances of diagnosis (year of diagnosis, symptoms, medical examination, fasting or random glucose concentrations at diagnosis), actual diabetes therapy (prescription of diet or physical activity, list of glucoselowering drugs taken) and most recent fasting glucose and $\mathrm{HbA}_{1 \mathrm{c}}$ levels.

Women were identified for validation if they had one of: (1) fasting plasma glucose $\geq 7.0 \mathrm{mmol} / 1$; random glucose $\geq 11.1 \mathrm{mmol} / 1$ at diagnosis; (2) report of glucose-lowering medication use; or (3) most recent fasting glucose or $\mathrm{HbA}_{1 \mathrm{c}}$ concentration $\geq 7.0 \mathrm{mmol} / 1$ or $\geq 7 \%$, respectively. For validation, results were cross-checked against at least two of: selfreported diabetes in the follow-up questionnaires; ascertained diabetes in the diabetes-specific questionnaire; or drug reimbursed by insurance. Women reimbursed at least twice for glucose-lowering medications were validated as having 
diabetes even when they did not answer the diabetes-specific questionnaire.

For administrative and logistic reasons, diabetes incident after 2008 has not yet been validated according to the algorithm used for diabetes before 2008. Women with diabetes occurring after 2008 have been identified exclusively from reimbursement for glucose-lowering medications and not from self-declaration, and they have not completed the diabetes-specific questionnaire. The principal analysis described here includes only women with diabetes that occurred before 2008 .

Dietary questionnaire In June 1993, a validated 208 item dietary questionnaire was sent to participants to assess the habitual diet of the previous year. Food and drink consumption throughout the day took French meal patterns into account, with questions for each meal occasion, snacks and aperitifs. The validity and reproducibility of the questionnaire have been previously described [21].

Mean daily nutrient intake was evaluated using a food composition table derived from the French food composition table of the French Information Center on Food Quality [22]. Since the food composition table did not include the antioxidant capacity of foods, the antioxidant capacity was assigned to each food item based on a previously published database [23]. Within this database the antioxidant capacity was estimated using two assays: the ferric ion-reducing antioxidant power (FRAP), based on the single-electron transfer method [24]; and the total radical-trapping antioxidant parameter (TRAP), based on the hydrogen atom transfer method. Because the correlation between FRAP and TRAP was high (Pearson $r=0.999$ ), only results obtained with the FRAP index are presented. Two values were available for four food items (apple, melon, beer and vinegar), so we used the average value. There was no exact match in the database for the following six foods, thus we used the value of a similar item, based on similarities between botanical groups and in vitamin $\mathrm{E}$ and $\mathrm{C}$ and polyphenol content: mirabelle plum (matched with loquat), lychee (matched with loquat), mango (matched with persimmon), watercress (matched with spinach), lemon (matched with lemon juice) and alcohol-free beer (matched with beer).

The total antioxidant capacity of the diet was calculated excluding the contribution of coffee. The Maillard products of the coffee-roasting process are the main contributors to the in vitro antioxidant capacity of coffee. However, their high molecular weight means the proportion absorbed through the intestinal mucosa remains unclear, and their role in cellular antioxidant defence is questioned [25, 26]. Furthermore, because coffee is rich in antioxidants, it would drive the association in the FRAP calculation and obscure any associations between other sources of FRAP and type 2 diabetes.
Statistical analysis Missing values were $<5 \%$ for all variables and were imputed from the median value. HR estimates and 95\% CI values were obtained using Cox proportional hazards models, with age as the time scale. The time at entry was age at the start of follow-up and the exit time was the age when participants were diagnosed with type 2 diabetes, died, were lost to follow-up or were censored at the end of the follow-up period, whichever occurred first.

The main exposure variable was the total antioxidant capacity, measured by the FRAP index and calculated excluding the contribution from coffee. The FRAP index was categorised by quintile, and the lowest quintile group was used as the reference category in the models. We first performed a univariate analysis (Model 1), and then adjusted for smoking status (never vs ever smoker), physical activity (metabolic equivalents [MET]-h/week, as a continuous variable), education level (fewer or more than 14 years of education), hypertension (self-reported or use of blood pressurelowering drugs: yes vs no), hypercholesterolaemia (self-reported blood cholesterol $>6.2 \mathrm{mmol} / \mathrm{l}$ or use of cholesterollowering drugs: yes vs no), family history of diabetes (yes vs no) (Model 2). Finally, Model 3 was as Model 2 but further adjusted for diet-related variables, which included energy intake (kJ/day) and alcohol intake (g ethanol/day). The model was corrected for adherence scores to the healthy dietary pattern and the western dietary pattern, both derived with principal components analysis (PCA), as previously described [27]. We further adjusted Model 3 for BMI $\left(\mathrm{kg} / \mathrm{m}^{2}\right)$. All covariates used for adjustment in the models were collected by a questionnaire attached to the 1993 dietary questionnaire. Only the 'family history of diabetes' variable was collected later, in 2005.

Spline regression was used to better characterise the shape of the association between total antioxidant capacity and type 2 diabetes risk. The reference value for estimating HRs and 95\% CI was chosen as the FRAP minimum value (1.03 mmol/ day) and four knots were used (the 20th, 40th, 60th and 80th percentiles of the distribution).

Finally, we tested the interaction between FRAP and the main potential confounders: total energy intake, coffee consumption, fruit and vegetable consumption, alcohol intake, score of adherence to a western dietary pattern and to a healthy dietary pattern (all divided in two categories according to the median value), smoking status and BMI ( $\left.<\mathrm{vs} \geq 25 \mathrm{~kg} / \mathrm{m}^{2}\right)$, and if the interaction test was statistically significant, we performed stratified analyses.

All statistical tests were two-sided and considered statistically significant if $p<0.05$. Data were analysed with the Statistical Analysis Systems software package, version 9.4 (SAS Institute, Cary, NC, USA).

Sensitivity analyses To evaluate associations with the overall total antioxidant capacity, we ran the models with the FRAP 
score including coffee, as a sensitivity analysis. We also evaluated the associations between the total antioxidant capacity and type 2 diabetes excluding women with diabetes that occurred in the first 5 years of follow-up, to investigate potential reverse causation bias. Finally, we included in the analysis all women who developed diabetes after 2008, identified exclusively from the drug reimbursement database. In total, an additional 503 women were reimbursed at least twice between 2008 and 2012.

\section{Results}

Study population characteristics Compared with women in the lowest quintile group of total antioxidant capacity, those in the highest quintile group had a higher energy intake, consumed less coffee, more fruit and vegetables, and drank more tea and wine (Table 1). Moreover, women in the highest quintile group of total antioxidant capacity were more frequently smokers and were more likely to have more years of education.

The food groups that contributed the most to the total antioxidant capacity were fruit (23\%), vegetables (19\%), alcoholic beverages $(15 \%)$ and hot beverages such as tea, chicory and hot chocolate (12\%).

Total antioxidant capacity and type $\mathbf{2}$ diabetes risk Higher levels of total antioxidant capacity were associated with lower risk of type 2 diabetes (Table 2). In particular, Model 3 highlighted that, in comparison with women in the lowest quintile group, women in the second, third, fourth and fifth quintile groups of total antioxidant capacity had HRs of 0.85 (95\% CI 0.74, 1.00), 0.70 (95\% CI 0.60, 0.73), 0.62 (95\% CI $0.52,0.73)$ and $0.61(95 \%$ CI $0.50,0.74)$, respectively (Table 2). The magnitude of this association decreased, but remained statistically significant, when further adjusted for BMI: 0.89 (95\% CI 0.76, 1.03), 0.74 (95\% CI 0.63, 0.86), 0.70 (95\% CI $0.59,0.83$ ) and 0.73 (95\% CI $0.60,0.89)$, respectively for the third, fourth and fifth quintile groups of the dietary total antioxidant capacity (Table 2).

All tests for interaction were not statistically significant, with the exception of coffee (test for interaction $p$ value $=0.02$ ). When stratifying the models on coffee consumption, the inverse association between total antioxidant capacity and type 2 diabetes was stronger for women with coffee consumption below the median consumption ( $247 \mathrm{ml} /$ day) (Table 3). When additionally adjusted for BMI, the association between total antioxidant capacity and type 2 diabetes was observed only in women with a lower coffee consumption (Table 3).

Using spline regression curves, there was a negative association between total antioxidant capacity and type 2 diabetes risk. More specifically, the inverse association between total antioxidant capacity and risk of type 2 diabetes was linear up to values of FRAP at around $15 \mathrm{mmol} /$ day, after which a plateau effect was reached (Fig. 1).

Sensitivity analyses When coffee was included in the total antioxidant capacity index, as measured by FRAP, the association between total antioxidant capacity and risk of developing type 2 diabetes was still statistically significant, although less strongly so. Indeed, HRs of 0.83 (95\% CI 0.71, 0.97), 0.83 (95\% CI $0.71,0.97)$ and 0.76 (95\% CI $0.65,0.89)$ were found for the third, fourth and fifth quintiles, respectively, compared with the first.

When excluding women with diabetes that occurred during the first 5 years of follow-up ( 365 women), the results obtained were equivalent to those of the main analyses in terms of magnitude and statistical significance (data not shown).

Finally, the results obtained when including the women with diabetes that occurred after 2008 and identified exclusively from the drug reimbursement database (503 women) did not differ from the main results (see electronic supplementary material [ESM] Table 1).

\section{Discussion}

In this cohort of French middle-aged women, higher total antioxidant capacity of the diet, measured excluding the contribution of coffee, was associated with a lower risk of type 2 diabetes. To our knowledge, this is the first prospective study to evaluate the risk of type 2 diabetes associated with total antioxidant capacity of the diet.

Previous studies have reported that the total antioxidant capacity is inversely associated with risk of the metabolic syndrome, abdominal obesity and hypertension in adults [28]. Similarly, in healthy young men and women, a linear inverse association between total antioxidant capacity with glucose and lipid biomarkers, as well as with central adiposity measurements, was highlighted [29].

Montonen et al [30] found a significant association between vitamin $\mathrm{E}$ intake, $\alpha$-tocopherol, $\gamma$-tocopherol, $\delta$ tocopherol and $\beta$-tocotrienol with reduced risk of type 2 diabetes, supporting the hypothesis that intake of antioxidants from the diet reduces the risk of type 2 diabetes. However, other compounds with antioxidant activity, such as vitamin C, flavonoids and lycopene, were found to have no impact on risk of diabetes when considered individually [30-34].

In our population study, women with higher levels of total antioxidant capacity (measured excluding the contribution of coffee), were characterised by a high consumption of fruit and vegetables and tea, which are known to be rich in antioxidant compounds, and have previously been associated with reduced risk of type 2 diabetes [3, 35, 36]. Our findings strengthen the hypothesis, already suggested by others [31], that the inverse association between type 2 diabetes and fruit 
Table 1 Characteristics of the study population $(n=64,223)$ according to quintiles of the dietary total antioxidant capacity (FRAP method) excluding the contribution from coffee; E3N-EPIC cohort, 1993-2008

\begin{tabular}{|c|c|c|c|c|c|}
\hline \multirow[t]{2}{*}{ Characteristic } & \multicolumn{5}{|c|}{ Total antioxidant capacity excluding the contribution of coffee (mmol/day) } \\
\hline & $\begin{array}{l}\leq 8.72 \\
(n=12,844)\end{array}$ & $\begin{array}{l}8.73-11.29 \\
(n=12,845)\end{array}$ & $\begin{array}{l}11.30-13.93 \\
(n=12,844)\end{array}$ & $\begin{array}{l}13.94-17.55 \\
(n=12,845)\end{array}$ & $\begin{array}{l}>17.55 \\
(n=12,845)\end{array}$ \\
\hline \multicolumn{6}{|l|}{ Birth generation } \\
\hline$<1930$ & $955(7)$ & $940(7)$ & $944(7)$ & $924(7)$ & $798(6)$ \\
\hline $1930-1934$ & $1569(12)$ & $1598(12)$ & $1665(13)$ & $1572(12)$ & $1508(12)$ \\
\hline $1935-1939$ & $2359(18)$ & $2404(19)$ & $2441(19)$ & $2457(19)$ & $2309(18)$ \\
\hline $1940-1944$ & $3037(24)$ & $3093(24)$ & $2996(23)$ & $3115(24)$ & $3245(25)$ \\
\hline$\geq 1945$ & $4924(38)$ & $4810(37)$ & $4799(37)$ & $4777(37)$ & $4984(39)$ \\
\hline Hypertension & $1985(15)$ & $1891(15)$ & $1937(15)$ & $1822(14)$ & $1828(14)$ \\
\hline Hypercholesterolaemia & $1769(14)$ & $1681(13)$ & $1726(13)$ & $1715(13)$ & $1669(13)$ \\
\hline Family history of diabetes & $1428(11)$ & $1441(11)$ & $1451(11)$ & $1415(11)$ & $1357(11)$ \\
\hline More than 14 years of education & $11,608(90)$ & $11,879(93)$ & $11,929(93)$ & $12,044(94)$ & $12,097(94)$ \\
\hline \multicolumn{6}{|l|}{ BMI $\left(\mathrm{kg} / \mathrm{m}^{2}\right)$} \\
\hline$<20$ & $2326(18)$ & $2004(16)$ & $1927(15)$ & $1926(15)$ & $1757(14)$ \\
\hline $20-24$ & $7864(61)$ & $8335(65)$ & $8363(65)$ & $8490(66)$ & $8571(67)$ \\
\hline $25-29$ & $2196(17)$ & $2085(16)$ & $2128(17)$ & $2039(16)$ & $2133(17)$ \\
\hline$\geq 30$ & $458(4)$ & $421(3)$ & $427(3)$ & $390(3)$ & $383(3)$ \\
\hline \multicolumn{6}{|l|}{ Smoking status } \\
\hline Non-smoker & $7875(61)$ & $7343(57)$ & $6928(54)$ & $6523(51)$ & $5713(45)$ \\
\hline Ex-smoker & $3533(28)$ & $3961(31)$ & $4261(33)$ & $4492(35)$ & $4865(38)$ \\
\hline Smoker & $1436(11)$ & $1541(12)$ & $1656(13)$ & $1830(14)$ & $2266(18)$ \\
\hline Age (years) & $51.35( \pm 6.68)$ & $51.46( \pm 6.63)$ & $51.56( \pm 6.66)$ & $51.52( \pm 6.61)$ & $51.17( \pm 6.45)$ \\
\hline Physical activity (MET-h/week) & $34.13( \pm 52.65)$ & $37.22( \pm 43.39)$ & $37.97( \pm 49.38)$ & $39.19( \pm 50.61)$ & $41.72( \pm 52.18)$ \\
\hline Energy intake (kJ/day) & $\begin{array}{l}7544.13 \\
( \pm 1793.89)\end{array}$ & $\begin{array}{l}8484.78 \\
( \pm 1897.82)\end{array}$ & $\begin{array}{l}9043.75 \\
( \pm 2013.80)\end{array}$ & $\begin{array}{l}9675.45 \\
( \pm 2136.22)\end{array}$ & $\begin{array}{l}10,836.14 \\
( \pm 2422.03)\end{array}$ \\
\hline Alcohol intake (g/day) & $3.17( \pm 5.61)$ & $6.08( \pm 7.76)$ & $8.96( \pm 9.63)$ & $12.29( \pm 12.52)$ & $18.70( \pm 19.91)$ \\
\hline Carbohydrate intake (g/day) & $195.91( \pm 58.81)$ & $218.80( \pm 63.39)$ & $231.02( \pm 66.46)$ & $244.06( \pm 70.3)$ & $262.91( \pm 77.8)$ \\
\hline Omega-3 intake (g/day) & $1.16( \pm 0.41)$ & $1.33( \pm 0.45)$ & $1.44( \pm 0.46)$ & $1.55( \pm 0.51)$ & $1.75( \pm 0.62)$ \\
\hline Adherence to western diet (score) & $-0.41( \pm 0.75)$ & $-0.24( \pm 0.82)$ & $-0.12( \pm 0.86)$ & $0.01( \pm 0.94)$ & $0.28( \pm 1.07)$ \\
\hline Adherence to healthy diet (score) & $-0.59( \pm 0.74)$ & $-0.26( \pm 0.84)$ & $-0.1( \pm 0.89)$ & $0.06( \pm 0.94)$ & $0.35( \pm 1.09)$ \\
\hline \multicolumn{6}{|l|}{ Consumption (/day) } \\
\hline Coffee (ml) & $315.00( \pm 265.12)$ & $300.01( \pm 256.79)$ & $290.10( \pm 253.99)$ & $280.03( \pm 253.27)$ & $261.03( \pm 257.81)$ \\
\hline Fruit and vegetables (g) & $334.53( \pm 151.77)$ & $430.67( \pm 180.93)$ & $468.97( \pm 205.43)$ & $501.95( \pm 231.61)$ & $555.77( \pm 276.97)$ \\
\hline Processed meat (g) & $17.73( \pm 17.29)$ & $19.54( \pm 18.15)$ & $20.80( \pm 18.22)$ & $21.81( \pm 19.34)$ & $24.45( \pm 21.42)$ \\
\hline Sugar-sweetened beverages (ml) & $19.71( \pm 68.13)$ & $21.43( \pm 61.65)$ & $23.97( \pm 74.50)$ & $27.59( \pm 65.53)$ & $27.59( \pm 72.11)$ \\
\hline Artificially sweetened beverages ( $\mathrm{ml}$ ) & $31.43( \pm 134.43)$ & $27.59( \pm 136.31)$ & $29.56( \pm 117.68)$ & $31.24( \pm 117.42)$ & $40.00( \pm 138.94)$ \\
\hline Tea (ml) & $57.14( \pm 101.55)$ & $120.10( \pm 150.48)$ & $220.34( \pm 192.07)$ & $300.81( \pm 243.99)$ & $471.43( \pm 398.74)$ \\
\hline Chocolate (g) & $4.93( \pm 7.94)$ & $7.14( \pm 10.11)$ & $10.14( \pm 12.76)$ & $12.89( \pm 16.14)$ & $19.21( \pm 28.56)$ \\
\hline Wine (ml) & $28.57( \pm 44.63)$ & $49.26( \pm 67.34)$ & $75.00( \pm 88.15)$ & $110.00( \pm 116.46)$ & $159.28( \pm 192.08)$ \\
\hline
\end{tabular}

Data shown are $n(\%)$ or mean $( \pm \mathrm{SD})$

and vegetable consumption, as well as between type 2 diabetes and tea, may be mediated by the synergistic effect of the antioxidant compounds present in these food items.

Women with higher levels of total antioxidant capacity also reported higher wine consumption. Consumption of high quantities of alcoholic beverages is known to be associated with a higher risk of diabetes, while moderate alcohol consumption is associated with a decreased risk compared with no consumption [37]. Moreover, only wine has been associated with a reduced risk of diabetes, with no association 
Table 2 Risk of type 2 diabetes according to quintile groups of dietary total antioxidant capacity (FRAP method), excluding the contribution of coffee; E3N-EPIC cohort, 1993-2008

\begin{tabular}{|c|c|c|c|c|c|c|}
\hline \multirow[t]{2}{*}{ Model } & \multicolumn{5}{|c|}{ Total antioxidant capacity (mmol/day) } & \multirow[t]{2}{*}{$p$ trend } \\
\hline & $\leq 8.72$ & $8.73-11.29$ & $11.30-13.93$ & $13.94-17.55$ & $>17.55$ & \\
\hline 1 & Ref & $0.95(0.82,1.10)$ & $0.85(0.74,0.99)$ & $0.80(0.69,0.93)$ & $0.94(0.82,1.09)$ & 0.21 \\
\hline 2 & Ref & $0.97(0.84,1.12)$ & $0.87(0.75,1.01)$ & $0.82(0.71,0.95)$ & $0.96(0.83,1.12)$ & 0.32 \\
\hline 3 & Ref & $0.85(0.74,1.00)$ & $0.70(0.60,0.73)$ & $0.62(0.52,0.73)$ & $0.61(0.50,0.74)$ & 0.0004 \\
\hline $3+\mathrm{BMI}$ & Ref & $0.89(0.76,1.03)$ & $0.74(0.63,0.86)$ & $0.70(0.59,0.83)$ & $0.73(0.60,0.89)$ & $<0.0001$ \\
\hline
\end{tabular}

Data are HR $(95 \% \mathrm{CI}) ;(n=64,223)$

Model 1: univariate analysis. Model 2: Model $1+$ smoking status (never smoker vs ever smoker), physical activity (MET-h/week), education level (less or more than 14 years of education), hypertension (self-reported or use of blood pressure-lowering drugs: yes vs no), hypercholesterolaemia (selfreported blood cholesterol $>6.2 \mathrm{mmol} / 1$ or use of cholesterol-lowering drugs: yes vs no), family history of diabetes (yes vs no). Model 3: Model $2+$ energy intake ( $\mathrm{kJ} / \mathrm{day})$, alcohol intake ( $\mathrm{g}$ ethanol/day), adherence score for the healthy dietary pattern and adherence score for the western dietary pattern

reported for beer or spirits [38, 39]. When the overall alcohol intake is moderate, the health benefits related to the abundant content of phenolic acids and polyphenols, present mainly in wine, with antioxidant activity prevail over the deleterious effect of the alcohol itself [40, 41].

Previous studies, including the E3N-EPIC study, have reported an inverse association between coffee consumption and risk of type 2 diabetes $[42,43]$. In our study, we did not include coffee in the calculation of total antioxidant capacity because there is no consensus on whether the antioxidants present in coffee are actually absorbed, and because coffee has such a high antioxidant content that it would drive the association with type 2 diabetes. Finally, because coffee consumption is often related to unhealthy behaviours, such as a stressful life, tobacco smoking and alcohol drinking, there could be some residual confounding. Our results confirm this hypothesis: when performing stratified analyses according to coffee consumption, the association between FRAP and type
2 diabetes disappeared in the group with high coffee consumption, suggesting that coffee consumption mediates the association with type 2 diabetes in this group. Similarly, when considering coffee in the FRAP calculation in sensitivity analyses, the inverse association with type 2 diabetes was still present but weakened. Indeed, the results obtained with the sensitivity analysis more likely reflected the association between type 2 diabetes and coffee, rather than with the overall total antioxidant capacity of the diet.

The spline regression curve shows how the inverse association between total antioxidant capacity and risk of type 2 diabetes reaches a plateau for values of FRAP above $15 \mathrm{mmol} /$ day. In a previous study, Cao et al reported a linear correlation between fasting plasma oxygen radical absorbance capacity and daily intake of total antioxidants, but when daily intake of total antioxidants was over $3.0 \mathrm{mmol}$, the fasting plasma oxygen radical absorbance capacity reached a plateau [44]. Indeed, in our study, a constant HR for FRAP values

Table 3 Risk of type 2 diabetes by quintile of dietary total antioxidant capacity stratified according to levels of coffee and alcohol consumption; E3NEPIC cohort, 1993-2008

\begin{tabular}{|c|c|c|c|c|c|c|}
\hline \multirow[t]{2}{*}{ Model } & \multicolumn{5}{|c|}{ Total antioxidant capacity (mmol/day) } & \multirow[t]{2}{*}{$p$ trenc } \\
\hline & $\leq 8.72$ & $8.73-11.29$ & $11.30-13.93$ & $13.94-17.55$ & $>17.55$ & \\
\hline \multicolumn{7}{|c|}{ Coffee consumption $<247 \mathrm{ml} /$ day } \\
\hline 3 & Ref & $0.72(0.59,0.88)$ & $0.69(0.56,0.83)$ & $0.56(0.44,0.69)$ & $0.53(0.41,0.67)$ & 0.0001 \\
\hline $3+\mathrm{BMI}$ & Ref & $0.77(0.63,0.93)$ & $0.70(0.57,0.85)$ & $0.62(0.50,0.77)$ & $0.61(0.48,0.78)$ & 0.003 \\
\hline \multicolumn{7}{|c|}{ Coffee consumption $>247 \mathrm{ml} /$ day } \\
\hline 3 & Ref & $1.03(0.83,1.28)$ & $0.66(0.54,0.89)$ & $0.66(0.50,0.86)$ & $0.68(50.49,0.93)$ & 0.013 \\
\hline $3+\mathrm{BMI}$ & Ref & $1.04(0.86,1.31)$ & $0.78(0.61,1.00)$ & $0.78(0.59,1.03)$ & $0.89(0.65,1.22)$ & 0.414 \\
\hline
\end{tabular}

Data are HR (95\% CI); $(n=64,223)$

Model 3: smoking status (never vs ever smoker), physical activity (MET-h/week, as continuous variable), education level (less or more than 14 years of education), hypertension (self-reported or use of blood pressure-lowering drugs: yes vs no), hypercholesterolaemia (self-reported blood cholesterol $>6.2 \mathrm{mmol} / \mathrm{l}$ or use of cholesterol-lowering drugs: yes vs no), family history of diabetes (yes vs no), energy intake (kJ/day), alcohol intake (g ethanol/ day), adherence score for the healthy dietary pattern, and adherence score for the western dietary pattern 


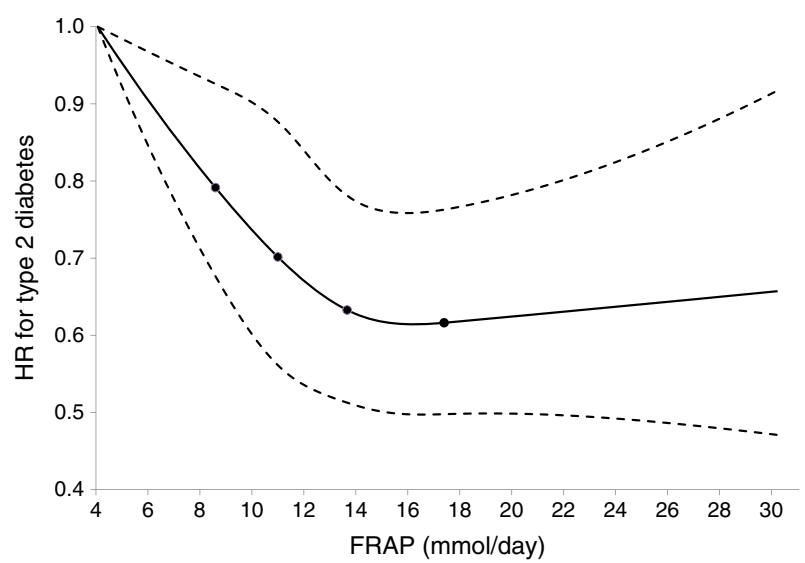

Fig. 1 Cubic spline regression model between the total antioxidant capacity (FRAP [mmol/day]) and HRs for type 2 diabetes; E3N-EPIC cohort, 1993-2008 $(n=64,223)$. Spline regression: the four knots are the 20th, 40th, 60th and 80th percentiles. The reference value is the minimum total antioxidant capacity. The model was adjusted for smoking status (never vs ever smoker), physical activity (MET-h/week, as a continuous variable), education level (less or more than 14 years of education), hypertension (self-reported or use of blood pressure-lowering drugs: yes vs no), hypercholesterolaemia (self-reported blood cholesterol $>6.2 \mathrm{mmol} / 1$ or use of cholesterol-lowering drugs: yes vs no), family history of diabetes (yes vs no), energy intake ( $\mathrm{kJ} /$ day), alcohol intake (g ethanol/day), adherence score for the healthy dietary pattern, adherence score for the western dietary pattern, and BMI $\left(\mathrm{kg} / \mathrm{m}^{2}\right)$. Solid line, HR; dashed lines, $95 \%$ CI

above $15 \mathrm{mmol} /$ day is in agreement with the findings of Cao et al, and it could therefore reflect, as suggested, saturation of the organism's capacity to absorb antioxidants from the diet. Undoubtedly, further studies are needed to clarify the underlying biological mechanism.

Strengths and limitations Potential limitations of the present study should be considered. The dietary estimates are based on a single questionnaire at baseline, thus misclassification of exposure is possible. However, as the study is prospective, any effects are likely to be non-differential and lead to an attenuation of the true association. Our study population included only women. This limitation should be minor, as few studies have reported differences in type 2 diabetes risk factors between men and women, although biological mechanisms may differ between sexes.

The E3N-EPIC cohort, as is the case for most general population cohorts, is not representative of the general population as it has a low proportion of socially deprived people and comprises, rather, health-conscious women. Therefore, the prevalence of overweight and obesity is lower compared with the general population, which probably explains the lower prevalence of diabetes. This reduces the potential value of extending the results to the general population in terms of attributable fraction of risk, but should not reduce the internal validity of the findings.

The algorithm used in the E3N-EPIC cohort to validate type 2 diabetes could be used for women only until 2008 .
Although this might represent a limitation, we are confident that the impact on the results is negligible due to the fact that the main analysis is based on 18 years of follow-up and has sufficient statistical power ( $n=1751$ women). Our confidence is strengthened by the fact that the results did not change when we included women with diabetes that occurred after 2008, identified exclusively from the drug reimbursement database.

The strengths of this study include the large sample size and the prospective design of the E3N-EPIC cohort, which allowed us to perform sensitivity analyses while keeping sufficient statistical power to detect associations, making the reverse causation hypothesis unlikely. We analysed women with validated type 2 diabetes only, based on a well-defined validation algorithm, which reduces the risk of false-negatives or false-positives. Finally, extensive information on potential confounders was collected, minimising residual confounding, and the dietary questionnaire used to assess the total antioxidant capacity intake was validated and specific to this French population.

Conclusions Our findings suggest that the total antioxidant capacity of the diet may play a role in reducing the risk of type 2 diabetes in middle-aged women. As type 2 diabetes represents a high disease burden worldwide, our results may have important public health implications. More studies are warranted to confirm our findings and better understand the biological mechanisms that underlie this inverse association.

Acknowledgements The authors are indebted to all participants for their continued participation. They are also grateful to all members of the $\mathrm{E} 3 \mathrm{~N}$ study group.

Data availability The datasets generated during and/or analysed for the current study are not publicly accessible, but are available from the corresponding author on reasonable request.

Funding This present work was supported by a grant from the World Cancer Research Fund (WCRF) and by a grant for the E4N study from the Agence Nationale de Recherche (ANR-10-COHO-0006 grant). The validation of women with potential diabetes was supported by the European Union (Integrated Project LSHM-CT-2006-037197 in the 6th European Community Framework Programme) InterAct project.

Duality of interest The authors declare that they have no conflict of interest associated with this manuscript.

Contribution statement FRM and GF conceived and designed the study and performed the statistical analysis. FRM drafted the original manuscript. All authors contributed to the interpretation of data discussed in the manuscript, revised the manuscript and approved its final version to be published. GF is the guarantor of this work and, as such, had full access to all the data in the study and takes responsibility for the integrity of the data and the accuracy of the data analysis. 


\section{References}

1. American Diabetes Association (2009) Diagnosis and classification of diabetes mellitus. Diabetes Care 32(Suppl 1):S62-S67

2. Ardisson Korat AV, Willett WC, Hu FB (2014) Diet, lifestyle, and genetic risk factors for type 2 diabetes: a review from the Nurses' Health Study, Nurses' Health Study 2, and Health Professionals' Follow-up Study. Curr Nutr Rep 3:345-354

3. Maritim AC, Sanders RA, Watkins JB 3rd (2003) Diabetes, oxidative stress, and antioxidants: a review. J Biochem Mol Toxicol 17: 24-38

4. Asmat U, Abad K, Ismail K (2016) Diabetes mellitus and oxidative stress - a concise review. Saudi Pharm J 24:547-553

5. Ceriello A (2000) Oxidative stress and glycemic regulation. Metabolism 49(Suppl 1):27-29

6. Dakhale GN, Chaudhari HV, Shrivastava M (2016) Supplementation of vitamin $\mathrm{C}$ reduces blood glucose and improves glycosylated hemoglobin in type 2 diabetes mellitus: a randomized, double-blind study. Saudi Pharm J 24:547-553

7. Manning PJ, Sutherland WH, Walker RJ et al (2004) Effect of highdose vitamin $\mathrm{E}$ on insulin resistance and associated parameters in overweight subjects. Diabetes Care 27:2166-2171

8. Rani AJ, Mythili SV (2014) Status in relation to oxidative stress in type 2 diabetes mellitus. J Clin Diagn Res 8:108-110

9. Qureshi SA, Lund AC, Veierød MB et al (2014) Food items contributing most to variation in antioxidant intake; a cross-sectional study among Norwegian women. BMC Public Health 14:45

10. Urquiza-Martínez MV, Fenton NB (2016) Antioxidant capacity of food. Free Radicals Antioxid 6:1

11. Carlsen M, Halvorsen BL, Holte K et al (2010) The total antioxidant content of more than 3100 foods, beverages, spices, herbs and supplements used worldwide. Nutr J 9:3

12. Brighenti F, Valtuena S, Pellegrini N et al (2005) Total antioxidant capacity of the diet is inversely and independently related to plasma concentration of high-sensitivity C-reactive protein in adult Italian subjects. Br J Nutr 93:619-625

13. Serafini M, Bellocco R, Wolk A, Ekström AM (2002) Total antioxidant potential of fruit and vegetables and risk of gastric cancer. Gastroenterology 123:985-991

14. Del Rio D, Agnoli C, Pellegrini N et al (2011) Total antioxidant capacity of the diet is associated with lower risk of ischemic stroke in a large Italian cohort. J Nutr 141:118-123

15. Bastide N, Dartois L, Dyevre V et al (2016) Dietary antioxidant capacity and all-cause and cause-specific mortality in the E3NEPIC/EPIC cohort study. Eur J Nutr 56:1233-1243

16. Kim K, Vance TM, Chun OK (2016) Greater Total antioxidant capacity from diet and supplements is associated with a less atherogenic blood profile in U.S. adults. Nutrients 8:15

17. Rautiainen S, Lindblad BE, Morgenstern R, Wolk A (2014) Total antioxidant capacity of the diet and risk of age-related cataract: a population-based prospective cohort of women. JAMA Ophthalmol 132:247-252

18. Clavel-Chapelon F, van Liere MJ, Giubout C et al (1997) E3NEPIC, a French cohort study on cancer risk factors. E3N-EPIC Group. Etude Epidemiologique aupres de femmes de l'Education Nationale. Eur J Cancer Prev 6:473-478

19. Clavel-Chapelon F, E3N Study Group (2015 Jun) Cohort profile: the French E3N cohort study. Int J Epidemiol 44:801-809

20. Consortium IA, Langenberg C, Sharp S et al (2011) Design and cohort description of the InterAct Project: an examination of the interaction of genetic and lifestyle factors on the incidence of type 2 diabetes in the EPIC Study. Diabetologia 54:2272-2282

21. van Liere MJ, Lucas F, Clavel F, Slimani N, Villeminot S (1997) Relative validity and reproducibility of a French dietary history questionnaire. Int J Epidemiol 26(Suppl 1):S128-S136
22. Agence Nationale de Securit_e Sanitaire (ANSES) [Internet]. Table de composition nutritionnelle des aliments Ciqual; 2013. Available from: www.ansespro.fr/TableCIQUAL/. Accessed 12 December 2016

23. Pellegrini N, Serafini M, Salvatore S, Del RD, Bianchi M, Brighenti F (2006) Total antioxidant capacity of spices, dried fruits, nuts, pulses, cereals and sweets consumed in Italy assessed by three different in vitro assays. Mol Nutr Food Res 50:1030-1038

24. Pellegrini N, Serafini M, Colombi B et al (2003) Total antioxidant capacity of plant foods, beverages and oils consumed in Italy assessed by three different in vitro assays. J Nutr 133:2812-2819

25. Serafini M, Jakszyn P, Luján-Barroso L et al (2012) Dietary total antioxidant capacity and gastric cancer risk in the European prospective investigation into cancer and nutrition study. Int J Cancer 15:131:E544-554

26. Priftis A, Stagos D, Konstantinopoulos K et al (2015) Comparison of antioxidant activity between green and roasted coffee beans using molecular methods. Mol Med Rep 12:7293-7302

27. Thorpe MG, Milte CM, Crawford D, McNaughton SA (2016) A comparison of the dietary patterns derived by principal component analysis and cluster analysis in older Australians. Int J Behav Nutr Phys Act 13:30

28. Bahadoran Z, Golzarand M, Mirmiran P, Shiva N, Azizi F (2012) Dietary total antioxidant capacity and the occurrence of metabolic syndrome and its components after a 3-year follow-up in adults: Tehran Lipid and Glucose Study. Nutr Metab (Lond) 9:70

29. Hermsdorff HH, Puchau B, Volp AC et al (2011) Dietary total antioxidant capacity is inversely related to central adiposity as well as to metabolic and oxidative stress markers in healthy young adults. Nutr Metab (Lond) 8:59

30. Montonen J, Knekt P, Jarvinen R, Reunanen A (2004) Dietary antioxidant intake and risk of type 2 diabetes. Diabetes Care 27: 362-366

31. Song Y, Manson JE, Buring JE, Sesso HD, Liu S (2005) Associations of dietary flavonoids with risk of type 2 diabetes, and markers of insulin resistance and systemic inflammation in women: a prospective study and cross-sectional analysis. J Am Coll Nutr 24:376-384

32. Nettleton JA, Harnack LJ, Scrafford CG, Mink PJ, Barraj LM, Jacobs DR Jr (2006) Dietary flavonoids and flavonoid-rich foods are not associated with risk of type 2 diabetes in postmenopausal women. J Nutr 136:3039-3045

33. Knekt P, Kumpulainen J, Jarvinen R et al (2002) Flavonoid intake and risk of chronic diseases. Am J Clin Nutr 76:560-568

34. Wang L, Liu S, Manson JE, Gaziano JM, Buring JE, Sesso HD (2006) The consumption of lycopene and tomato-based food products is not associated with the risk of type 2 diabetes in women. $\mathrm{J}$ Nutr 136:620-625

35. Li M, Fan Y, Zhang X, Hou W, Tang Z (2014) Fruit and vegetable intake and risk of type 2 diabetes mellitus: meta-analysis of prospective cohort studies. BMJ Open 4:e005497

36. Yang WS, Wang WY, Fan WY, Deng Q, Wang X (2014) Tea consumption and risk of type 2 diabetes: a dose-response meta-analysis of cohort studies. Br J Nutr 111:1329-1339

37. Carlsson S, Hammar N, Grill V (2005) Alcohol consumption and type 2 diabetes meta-analysis of epidemiological studies indicates a U-shaped relationship. Diabetologia 48:1051-1054

38. Zenebe W, Pechánová O, Bernátová I (2001) Protective effects of red wine polyphenolic compounds on the cardiovascular system. Exp Clin Cardiol 6:153-158

39. Arranz S, Chiva-Blanch G, Valderas-Martínez P, Medina-Remón A, Lamuela-Raventós RM, Estruch R (2012) Wine, beer, alcohol and polyphenols on cardiovascular disease and cancer. Nutrients 4 : 759-781 
40. Howard AA, Arnsten JH, Gourevitch MN (2004) Effect of alcohol consumption on diabetes mellitus: a systematic review. Ann Intern Med 140:211-219

41. Hodge AM, English DR, O’Dea K, Giles GG (2006) Alcohol intake, consumption pattern and beverage type, and the risk of type 2 diabetes. Diabet Med 23:690-697

42. Sartorelli DS, Fagherazzi G, Balkau B et al (2010) Differential effects of coffee on the risk of type 2 diabetes according to meal consumption in a French cohort of women: the E3N-EPIC/EPIC cohort study. Am J Clin Nutr 91:1002-1012

43. van Dam RM, Willett WC, Manson JE, Hu FB (2006) Coffee, caffeine, and risk of type 2 diabetes: a prospective cohort study in younger and middle-aged U.S. women. Diabetes Care 29:398-403

44. Cao G, Booth SL, Sadowski JA, Prior RL (1998) Increases in human plasma antioxidant capacity after consumption of controlled diets high in fruit and vegetables. Am J Clin Nutr 68:1081-1087 